\title{
Histopathology of Surgically Resected Fallopian Tubes with Special Emphasis on Serous Tubal Intraepithelial Carcinoma
}

\author{
Rallapalli Rajyalakshmi ${ }^{1 *}$, Raparthi Srujana ${ }^{2}$ and Rani Vijaya Bhaskar ${ }^{1}$ \\ ${ }^{1}$ Department of Pathology, Rangaraya Medical College, Kakinada, 533001 India \\ ${ }^{2}$ Department of Pathology, Andhra Medical College, Visakhapatnam, India
}

\begin{abstract}
Background: The fallopian tube, though very common surgical specimen is rarely studied. It is affected by a spectrum of lesions ranging from inflammatory to rare neoplasms. Recent studies suggest a precursor lesion for high grade serous carcinoma of the ovary in the fallopian tube mucosa and is known as serous tubal intraepithelial carcinoma. In the present study, we analyzed the histopathology of fallopian tube lesions including serous tubal intraepithelial carcinoma.
\end{abstract}

Methods: All the fallopian tube specimens received were grossed according to protocol and were routinely processed and stained. Atypical proliferative lesions of mucosa were subjected to immunohistochemical markers p53 and ki67.

Result: Out of 350, 106 specimens showed a spectrum of lesions, of which hydrosalpinx was the commonest. Rest of the lesions were chronic and acute salpingitis, tuberculous salpingitis and tubal endometriosis. We encountered a single case of primary serous carcinoma of the fallopian tube. Immunohistochemistry on ten cases of atypical mucosal proliferations confirmed a single case each of serous tubal intraepithelial carcinoma and serous tubal intraepithelial lesion.

Conclusion: Though fallopian tube is unremarkable in most cases, thorough sampling can detect significant pathological lesions including precursor lesions.

Keywords: Fallopian Tube, Serous Tubal Intraepithelial Carcinoma, Serous Tubal Intraepithelial Lesion, Serous Adenocarcinoma

\section{Introduction}

The fallopian tube is a very common surgical specimen in the pathology laboratory.

Various tubal disorders include common lesions like hydrosalpinx to rare primary carcinomas. Inflammatory diseases of the tube are the commonest and are responsible for infertility. ${ }^{[1,2,3]}$ There were very few studies about the histological findings of the fallopian tube. As this is a common specimen, knowledge of these lesions can be valuable to the pathologist.

Traditionally, serous carcinoma of the ovary was thought to arise from the surface epithelium and epithelial inclusion cysts of the ovary. During the last few years, a precursor lesion was recognized in the fallopian tube, especially within the fimbrial end and is known as "serous tubal intraepithelial carcinoma" (STIC). ${ }^{[4,5]}$ Accordingly, STIC is probably the earliest histologically recognizable lesion in the pathogenesis of serous neoplasms including both low grade and high-grade serous carcinoma. Therefore, routine histological examination of the fimbria provides an opportunity to detect these early malignant changes. ${ }^{[6,7]}$

With this concept in mind, we take up the study of the spectrum of histopathological lesions of the fallopian tube including serous tubal intraepithelial carcinoma.

\section{Aims and objectives:}

1) To study histopathological features of surgically resected specimens of fallopian tubes.

2) To identify and describe serous tubal intraepithelial carcinoma of the fallopian tubes.

3) To assess its relationship with serous neoplasms of the ovary.

\section{Materials and Methods}

The present study was a prospective study done at the Department of Pathology, Rangaraya Medical College, Kakinada for a period of two years, i.e from December 2014 to December 2016. The fallopian tubes obtained either by salpingectomy, salpingo-ophorectomy or along with hysterectomy were analyzed. All the specimens received were fixed in formalin for 24-48 hours. After fixation, a thorough gross examination was done according to protocol. A minimum number of 3 representative bits were taken from each tube. Two longitudinally cut sections from the fimbrial end and one transverse section from the remaining tube were taken and processed routinely to obtain paraffin sections of 4-5 microns thick and were stained with Haematoxylin \& Eosin. In suspected carcinoma of the ovary, grossing was done by SEE-FIM 
protocol. Immunohistochemistry was done for atypical proliferative mucosal lesions of fallopian tube.

Inclusion Criteria: Hysterectomy with salpingooophorectomy, salpingo-oophorectomy, and salpingectomy specimens.

Exclusion Criteria: Tubectomy done for sterilization and ectopic tubal gestation.

\section{Results}

A total number of 350 fallopian tube specimens were studied, of which majority, i.e 228/350 (65.1\%) were along with hysterectomy specimens. Remaining 120 cases $(34.2 \%)$ were unilateral or bilateral salpingooophorectomy specimens and a small number of (2/350) isolated salpingectomy specimens. The age distribution was from 11-70 years with the peak in the third decade.

Out of the total 350 cases, the majority i.e. 244/350 (69.7\%) did not show any significant abnormality on histopathology. Most of these were a part of TAH with BSO specimens. The remaining 106/350 (30.2\%) cases showed a spectrum of lesions as depicted in table 1 .

Most common lesions in the present study were inflammatory, of which hydrosalpinx was the commonest, followed by chronic and acute salpingitis. The two cases of tuberculous salpingitis were tubo-ovarian masses, which on microscopy showed classical confluent granulomas of tuberculosis. (Fig1) A single case of xanthogranulomatous salpingo-ophoritis revealed diffuse infiltration of mucosa with foamy histiocytes and lymphocytes.

A single case of endometriosis showed the presence of endometrial glands and stroma in the wall of the tube. (Fig 2)

We diagnosed a single case of metaplastic papillary tumor as an incidental finding on routine histopathological examination. Microscopy showed an exophytic papillary tumor involving part of the circumference of the mucosa. The epithelium was made up of non-ciliated columnar cells arranged in a single layer. (Fig 3)

Proliferative epithelial lesions are a group of lesions, which include adenomatous hyperplasia, atypical hyperplasia, carcinoma in situ and intraepithelial carcinoma.

The morphological criteria of Serous tubal intraepithelial carcinoma (STIC) proposed by Vang et al are, ${ }^{[8]}$

\section{H\&E criteria are}

1. Cellular crowding/ Stratification/ Loss of polarity

2. Nuclear enlargement, Hyperchromasia, irregular chromatin, prominent nucleoli

3. Mitosis, loss of cilia

\section{Immunohistochemical criteria are}

1. Aberrant $\mathrm{p} 53$ expression

2. Aberrant MIB-1 expression

Presence of either strong nuclear staining or complete absence of staining was considered as the aberrant p53 expression. Aberrant MIB-1 expression was increased nuclear staining in at least $10 \%$ of atypical cells, compared to that of the adjacent normal mucosa.

Atypical mucosal proliferative lesion similar to the STIC on H\&E, with either p53 or MIB-1 aberrant expression, was classified as the serous tubal intraepithelial lesion. (STIL)

If neither p53 nor MIB-1 was aberrant, the lesion was considered to be a non-neoplastic atypical mucosal proliferation of unknown significance. Ten cases in the present study showed abnormal mucosal proliferation of the fallopian tube on H\&E, which prompted us for immunohistochemical work-up.

Two out of these 10 cases showed cellular crowding, nuclear enlargement, hyperchromasia, atypical nuclear contours, and mitoses of the mucosa. One section each from these tubes was subjected to immunohistochemistry. One of the two cases showed aberrant immune expression of both p53 and MIB-1 and was diagnosed as serous tubal intraepithelial carcinoma. (Fig 4) Gross and microscopic examination of bilateral ovaries and the other side tube were normal.

Whereas the other case showed aberrant P53 expression only with low Ki-67 expression and hence was diagnosed as a serous tubal intraepithelial lesion. (Fig 5) The patient was a 11 years old female child, who presented with abdominal distension. Ultrasound showed a solid ovarian mass, which on microscopy, found to be juvenile granulosa cell tumor. Remaining eight cases with atypical mucosal proliferation did not demonstrate aberrant expression of either P53 or MIB-1 and were diagnosed as non-neoplastic atypical mucosal proliferation of unknown significance. The details of these proliferative lesions were shown in table 2.

We encountered a single case of primary papillary serous carcinoma of the fallopian tube. The patient was a 44 years old female who presented with vaginal discharge. We received hysterectomy with bilateral salpingooophorectomy. The involved tube was dilated and cut section of which showed $8 \times 3 \times 3 \mathrm{~cm}$ solid gray-white growth. Histopathology showed the features of papillary serous carcinoma. [Fig: 6A,B] Microscopy of the uterus, cervix and other side fallopian tube did not show any abnormality. 
A case of high-grade papillary serous cystadenocarcinoma of ovary infiltrating the tubal wall was noted. Two cases in the present study showed tumor emboli in the tubal lumen, the primary being ovarian papillary serous cystadenocarcinoma. In another case, a mixed germ cell tumor of the ovary infiltrated the tubal wall.

Table 1: Histopathology of spectrum of fallopian tube lesions.

\begin{tabular}{|c|c|c|}
\hline S.N0 & Diagnosis & No. of cases \\
\hline 1. & Hydrosalpinx & $49(46.23 \%)$ \\
\hline 2. & Chronic Salpingitis & $16(15.1 \%)$ \\
\hline 3. & Paratubal cysts & $16(15,1 \%)$ \\
\hline 4. & Hematosalpinx & $5(4.72 \%)$ \\
\hline 5. & Tubal endometriosis & $1(0.94 \%)$ \\
\hline 6. & Metaplastic papillary tumor & $1(0.94 \%)$ \\
\hline 7. & Tumor emboli & $2(1.9 \%)$ \\
\hline 8. & Atypical mucosal proliferation & $8(7.55 \%)$ \\
\hline 9. & STIL & $1(0.94 \%)$ \\
\hline 10. & STIC & $1(0.94 \%)$ \\
\hline 11. & Primary papillary serous carcinoma & $1(0.94 \%)$ \\
\hline 12. & Miscellaneous & $5(4.72 \%)$ \\
\hline 13. & Total & 106 \\
\hline
\end{tabular}

STIC: Serous tubal intraepithelial carcinoma, STIL: Serous tubal intraepithelial lesion

Table 2: Proliferative lesions of fallopian tube.

\begin{tabular}{|c|c|c|c|c|c|}
\hline S.No & Age in years & Histopathology of ovaries & P53 & Ki67 & Diagnosis \\
\hline 1 & 40 & Nil particular & + & + & STIC \\
\hline 2 & 11 & Juvenile granulosa cell tumour & + & - & STIL \\
\hline 3 & 34 & Mucinous cystadenoma & - & - & Atypical mucosal proliferation \\
\hline 4 & 34 & Mucinous cystadenoma & - & - & Atypical mucosal proliferation \\
\hline 5 & 55 & Nil particular & - & - & Atypical mucosal proliferation \\
\hline 6 & 50 & Nil particular & - & - & Atypical mucosal proliferation \\
\hline 7 & 40 & Nil particular & - & - & Atypical mucosal proliferation \\
\hline 8 & 35 & Nil particular & - & - & Atypical mucosal proliferation \\
\hline 9 & 40 & Serous cystadeno carcinoma & - & - & Atypical mucosal proliferation \\
\hline 10 & 35 & Serous cystadenocarcinoma & - & - & Atypical mucosal proliferation \\
\hline
\end{tabular}

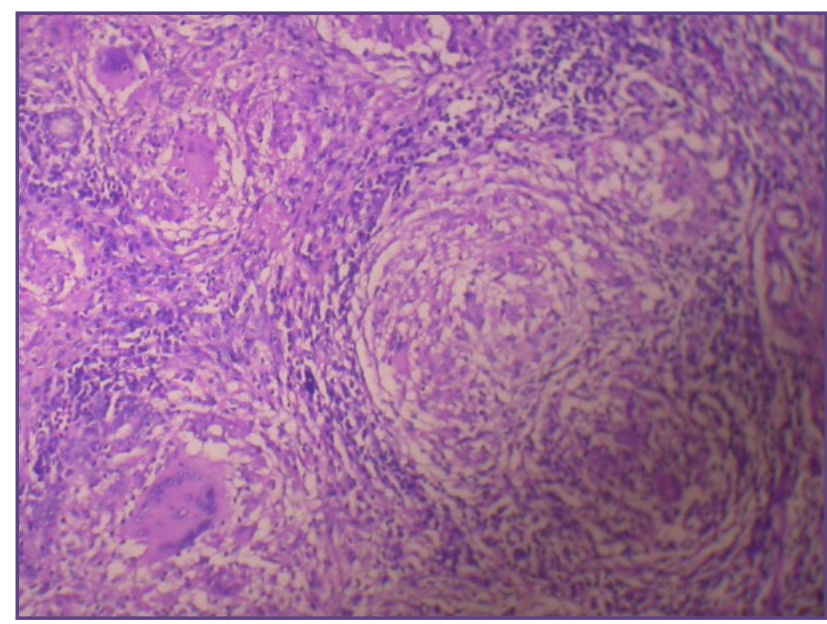

Fig. 1: Multiple granulomas in Tuberculosis of Fallopian tube (H\&E, 20X).

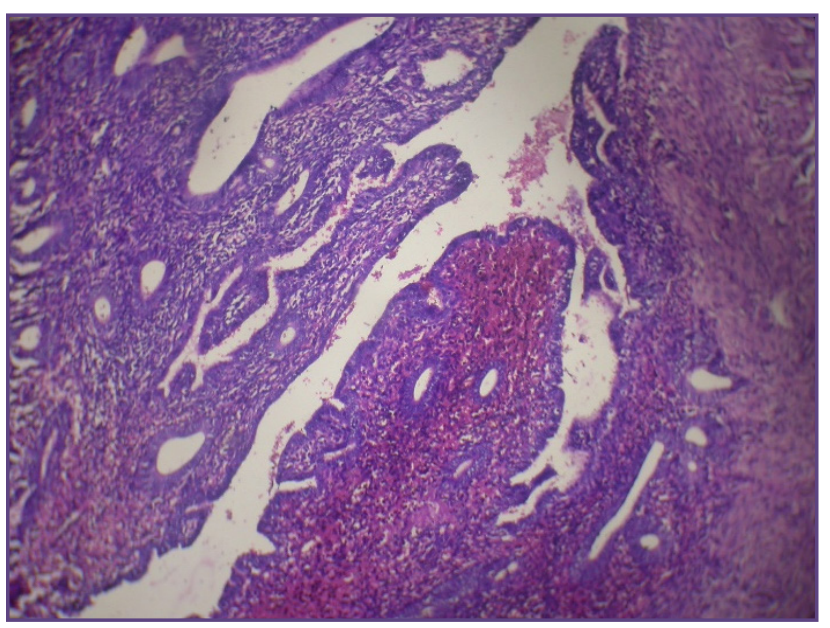

Fig. 2: Endometriosis of Fallopian tube. (H\&E, 20X). 


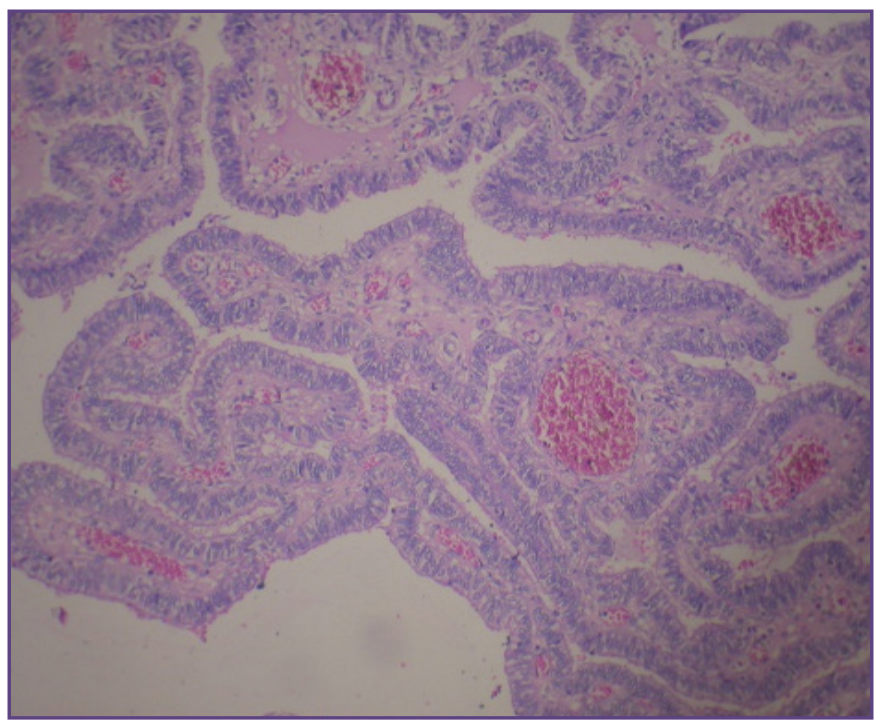

Fig. 3: Metaplastic papillary tumor of Fallopian tube (H\&E, 20X).

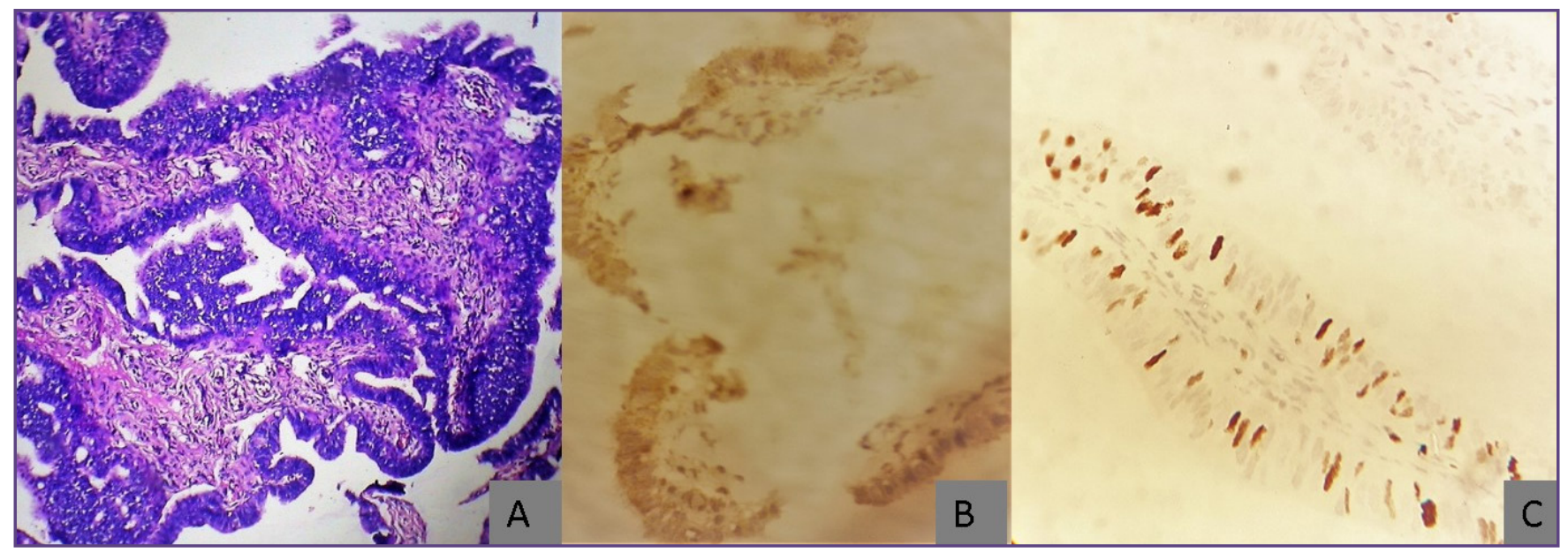

Fig. 4: H\&E (A), P53(B) and ki67(B) in a case of Serous tubal intraepithelial carcinoma.

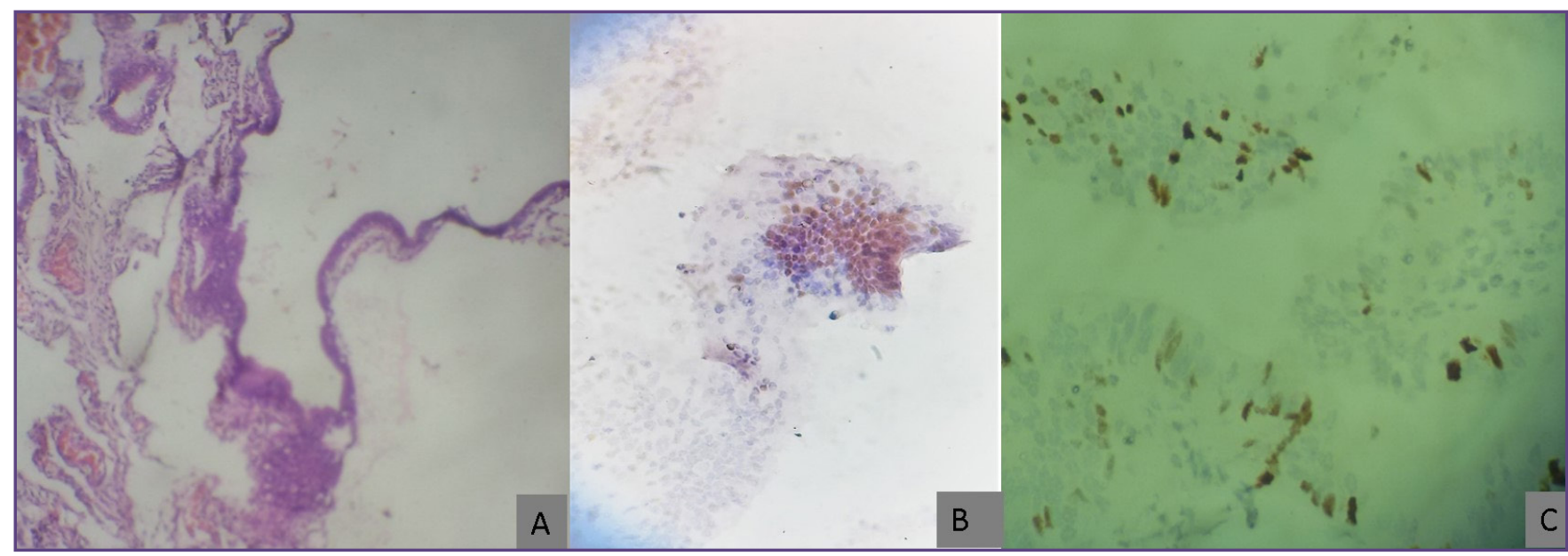

Fig. 5: H\&E(A) P53(B) and Ki67 (B) in a case of Serous intraepithelial lesion. 


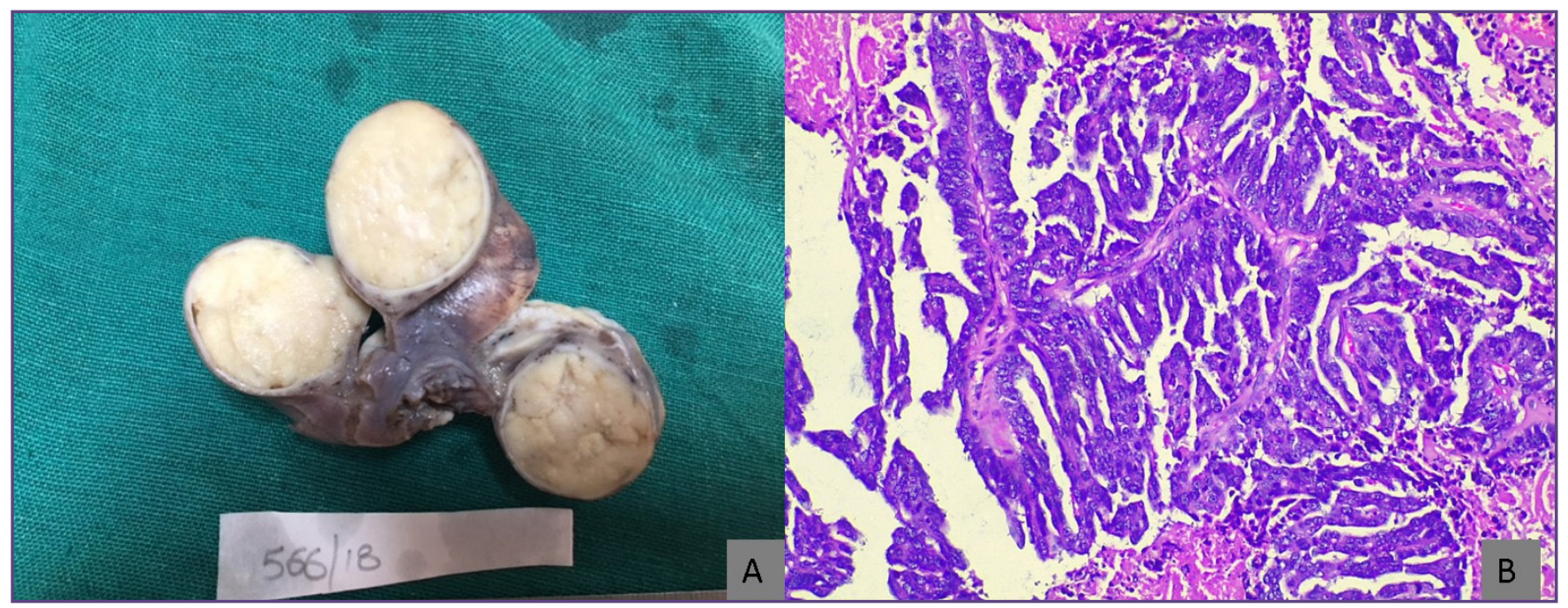

Fig. 6: Gross(A) and microscopy(B) of primary papillary serous carcinoma of Fallopian tube (H\&E, 20x).

\section{Discussion}

Like in other Indian studies, the majority of fallopian tube lesions in the present study were inflammatory in nature. The fallopian tube is a common site for tuberculosis of the female genital tract and is usually secondary to either pulmonary or extra-pulmonary tuberculosis. Tuberculosis along with other inflammatory diseases of the tube is an important cause of infertility. ${ }^{[9]}$

Endometriosis frequently involves the fallopian tube. A diagnosis of endometriosis requires the presence of endometrial glands and or endometrial stroma within the fallopian tube wall.

Both benign and malignant tumors can occur in the fallopian tube but are very uncommon.

The metaplastic papillary tumor is an uncommon pregnancy related papillary lesion with bland nuclear features. The neoplastic nature of this lesion is controversial. ${ }^{[10]}$

We got a single case of primary papillary serous carcinoma of the fallopian tube with $0.9 \%$ incidence, which is in comparison with the studies by Kasa Lakshmi et al [11] $(0.12 \%)$ and Gon S et al. ${ }^{[12]}(0.03 \%)$.

Primary carcinoma of the fallopian tube is an extremely uncommon tumor constituting $0.3-1.1 \%$ of all gynecological malignancies. ${ }^{[13]}$ However, the exact frequency is difficult to determine as advanced carcinomas of the tube usually involve ovary and resemble primary ovarian carcinoma. The clinical and histological features of primary tubal cancers are similar to that of ovarian cancers. They usually arise in the ampulla and causes fusiform swelling of the tube.

The criteria to consider a carcinoma of the fallopian tube as primary are,
1. Location of the tumor entirely within the tube

2. Absence of the same type of carcinoma in uterus and ovary. ${ }^{[13]}$

The entire spectrum of surface epithelial tumors of the ovary can occur, with serous being the commonest, constituting $50 \%$ of fallopian tube carcinomas. The morphology of serous carcinoma of the fallopian tube is similar to that of the ovary. Elevated CA 125 levels are an important marker. [14]

Most of the patients with primary serous adenocarcinoma of fallopian tube present with stage 1 disease at diagnosis, but their survival probability is low compared with other early-stage gynecological malignancy. A single case of high grade papillary serous cystadenocarcinoma of the ovary involved the tubal wall, where we could not establish the primary with certainty.

According to recent studies, mucosa of the fallopian tube, particularly of the fimbria is the site for the precursor lesion of familial high-grade serous carcinoma of the ovary. ${ }^{[15]}$ This precursor lesion is a non-invasive high-grade serous carcinoma, otherwise known as STIC.

Joseph T Rabban et al proposed the presence of a similar type of precursor lesion in the fimbria for sporadic cases. In their study, they found four STIC lesions out of 255 resected fallopian tubes of low-risk women. ${ }^{[16]}$

Diagnosis of STIC requires a stringent grossing protocol and unique histopathological and immunohistochemical criteria. "Sectioning and extensively examining the fimbriated end" or simply SEE-Fim grossing protocol was originally developed for BRCA mutation-positive highrisk patients. This procedure involves entire submission 
of the fallopian tube, which includes longitudinal sections of fimbria and transverse sections of the rest of the tube. $[17,18,19]$

Vang et al proposed the histopathology and immunohistochemistry criteria for the diagnosis of STIC. ${ }^{[8]}$ In the present study, we identified a single case each of serous tubal intraepithelial lesion (STIL) and serous tubal intraepithelial carcinoma (STIC) using the above criteria.

The present study included 9 cases of serous cystadenocarcinoma of the ovary. We used SEE-FIM protocol for gross examination of the fallopian tubes in these cases. Two of these nine cases showed tumor emboli in the lumen of the fallopian tube, one of the cases showed tumor infiltration of the tubal wall and two cases showed atypical mucosal proliferation. These two cases of atypical mucosal proliferation were followed up with p53 and Ki67 markers, which showed no aberrant expression. The remaining 4 cases showed no microscopic pathology.

Nine cases of serous carcinoma of the ovary in the present study do not associate with any proliferative lesions like STIC or STIL. Whereas, other studies reported a small percentage of STIC lesions, varying from 0.8 to $4 \%{ }^{[20]}$

The relatively higher percentage of STIC and STIL in other studies could be due to the larger sample size and highly selective sampling. Most of these studies were done on BRCA mutation positive patients. ${ }^{[20]}$ Whereas, our study was done on a random group, in whom the BRCA status was not known.

The major limitation to the tubal origin theory of serous carcinoma of the ovary is the absence of STIC lesions in a significant number of patients with high-grade serous carcinoma. ${ }^{[21]}$ In occasional case reports, borderline and low-grade serous carcinomas progressed to high-grade serous carcinomas. ${ }^{[22]}$ Other causes could be lack of awareness and experience of the pathologist. ${ }^{[23]}$

We followed SEE-FIM protocol method of grossing of the tube only for high-grade serous carcinoma of ovary due to limited resources. Embedding fimbria alone can detect most tubal lesions but not all STICs.

Financial constraints and selection bias for immunohistochemistry profiling are the limitations in the present study. Small sample size and inadequate sampling of the specimens could be other/additional factors.

\section{Conclusion}

In conclusion, though the fallopian tube remains unremarkable in the majority of the cases, it can harbor significant pathological lesions like tuberculosis, salpingitis, endometriosis which has a profound effect on the female fertility. Thorough sampling of the fallopian tube as per the protocol, followed by a meticulous examination of tubal mucosa can detect serous tubal intraepithelial carcinoma, which is the precursor of high grade pelvic serous carcinoma. Similar to the present study, a significant subset of high-grade serous carcinomas is not associated with STIC, proposing a non-tubal origin of high-grade serous carcinoma. Further studies are required to elucidate the etiologic significance of STIC in pelvic serous carcinogenesis. Though the fallopian tubes remain unremarkable in the majority of the surgical pathology specimens, it must be subjected for histopathological examination to demonstrate occult pathological lesions.

\section{Abbreviations}

STIC: Serous tubal intraepithelial carcinoma

STIL: Serous tubal intraepithelial lesion

H\&E: Hematoxylin and Eosin

\section{Reference}

1. Hunt JL, Lynn AA. Histologic features of surgically removed fallopian tubes. Arch Pathol Lab Med 2002; 126:951-55.

2. Bagwan IN, Harke MR, Malpani MR, Deshmukh SD. Histopathological study of spectrum of lesions encountered in the fallopian tube. J Obstet Gynecol Ind 2004; 54: 379-82.

3. Patel J, Iyer RR. Spectrum of histopathological changes in fallopian tubes-a study of 350 cases. International Journal of Scientific Research. 2016 18;5(1).

4. Kurman RJ, Shih IM. The Origin and pathogenesis of epithelial ovarian cancer-a proposed unifying theory. Am J Surg Pathol. 2010;34(3):433-43.

5. Hu CY, Taymor ML, Hertig AT. Primary carcinoma of the fallopian tube. Am J Obstet Gynecol.1950 Jan 1;59(1):5867.

6. Carlson JW, Miron A, Jarboe EA, et al. Serous tubal intraepithelial carcinoma: its potential role in primary peritoneal serous carcinoma and serous cancer prevention. J Clin Oncol. 2008 Sep 1;26(25):4160-65.

7. Semmel DR, Folkins AK, Hirsch MS, Nucci MR, Crum $\mathrm{CP}$. Intercepting early pelvic serous carcinoma by routine pathological examination of the fimbria. Mod Pathol. 2009 Aug 1;22(8):985-88.

8. Vang R, Visvanathan $\mathrm{K}$, Gross A, Maambo $\mathrm{E}$ et al. Validation of an algorithm for the diagnosis of serous tubal intraepithelial carcinoma. Int J Gynecol Pathol. 2012 May;31(3):243.

9. Grace GA, Devaleenal DB, Natrajan M. Genital tuberculosis in females. Indian J Med Res. 2017 Apr;145(4):425.

10. Jang MI, Sung JY, Kim JY, Kim HS. Clinicopathological characteristics of metaplastic papillary tumor of the fallopian tube. Anticancer Res. 2017 Jul 1;37(7):3693-701.

11. Lakshmi K, Baleswari G, Mallikarjun C, Arasi T, Rao L. Histopathological study of spectrum of lesions in the 
fallopian tubes. Journal of evolution of medical and dental sciences.2015 Jun;1(4):350-5.

12. Gon S, Basu A, Majumdar B, Das TK, Sengupta M, Ghosh D. Spectrum of histopathological lesions in the fallopian tubes. Journal of pathology of Nepal. 2013 Jan 1;3(5):35660 .

13. Kurman RJ, Carcangiu ML, Herrington CS, Young RH. WHO classification of tumors of Female reproductive organs 4th Edition, Lyon, World Health organization; 2014

14. Nag D, Bhaumik P, Nandi A, Samaddar A. Bilateral primary fallopian tube papillary serous carcinoma in postmenopausal woman: report of two cases. J midlife health. 2016 Jan;7(1):34.

15. Colgan TJ, Murphy J, Cole DE, Narod S, Rosen B. Occult carcinoma in prophylactic oophorectomy specimens: prevalence and association with BRCA germline mutation status. Am J Surg Pathol. 2001 Oct 1;25(10):1283-9.

16. Rabban JT, Garg K, Crawford B, Chen LM, Zaloudek CJ. Early detection of high-grade tubal serous carcinoma in women at low risk for hereditary breast and ovarian cancer syndrome by systematic examination of fallopian tubes incidentally removed during benign surgery. The Am J Surg Pathol. 2014 Jun 1;38(6):729-42.

17. Lee Y, Medeiros F, Kindelberger D, Callahan MJ, Muto MG, Crum CP. Advances in the recognition of tubal intraepithelial carcinoma: applications to cancer screening and the pathogenesis of ovarian cancer. Adv Anat Pathol. 2006 Jan 1;13(1):1-7.

18. Gwin K, Wilcox R, Montag A. Insights into selected genetic diseases affecting the female reproductive tract and their implication for pathologic evaluation of gynecologic specimens. Arch Pathol Lab Med. 2009 Jul;133(7):1041-52.

19. Mingels MJ, Van Ham MA, De Kievit IM, Snijders MP, Van Tilborg AA, Bulten J, Massuger LF. Müllerian precursor lesions in serous ovarian cancer patients: using the SEE-Fim and SEE-End protocol. Mod Pathol. 2014 Jul;27(7):1002.

20. Mahe E, Tang S, Deb P, Sur M, Lytwyn A, Daya D. Do deeper sections increase the frequency of detection of serous tubal intraepithelial carcinoma (STIC) in the "sectioning and extensively examining the FIMbriated end"(SEE-FIM) protocol?. Int J Gynecol Pathol. 2013 Jul 1;32(4):353-7.

21. Przybycin CG, Kurman RJ, Ronnett BM, Shih IM, Vang R. Are all pelvic (nonuterine) serous carcinomas of tubal origin?. Am J Surg Pathol. 2010 Oct 1;34(10):1407-16.

22. Li J, Fadare O, Xiang L, Kong B, Zheng W. Ovarian serous carcinoma: recent concepts on its origin and carcinogenesis. J Hematol Oncol. 2012 Dec;5(1):8.

23. Ferguson DC, Han LM, Wang Y et al. The role of the fallopian tube in ovarian serous carcinogenesis: biologic mechanisms and clinical impacts. Am J Clin Exp Obstet Gynecol. 2015;2(1):1-3.

*Corresponding author:

Dr. R.Rajyalakshmi, Associate professor, Department of pathology, Opposite Government General Hospital, Rangaraya Medical College,

Kakinada-533001

Phone: +919848293673

Email: rrlakshmi09@gmail.com

Financial or other Competing Interests: None. 